\title{
Integrated Raman- and Angular-Scattering Microscopy
}

\author{
Zachary J. Smith and Andrew J. Berger \\ The Institute of Optics, University of Rochester, \\ Rochester, NY 14627
}

\begin{abstract}
A microscopy system has been constructed which is capable of simultaneously acquiring both traditional Raman spectra as well as angle-resolved elastic scattering patterns using a single focused laser spot less than $10 \mu \mathrm{m}$ wide. The elastic-scattering signal was analyzed by Generalized Lorenz-Mie theory, representing the first experimental validation of the theory's prediction of angular backscatter from single spheres. The microscope system exhibits $3 \mathrm{~nm}$ precision in predicting sphere diameters, while simultaneously yielding high quality Raman signals. Applications to single cell analysis are envisioned. (c) 2008 Optical Society of America
\end{abstract}

OCIS codes: $170.5660,290.4020$

Raman spectroscopy of microscopic samples is an important research tool for investigating questions in cell biology [1]. The chief advantage of Raman spectroscopy of single cells over more traditional methods such as fluorescence tagging is that Raman spectra are endogenous signals, requiring no external labelling or sample preparation. This allows cells to retain their full functionality, for example during immune cell activation [1]. A typical molecule will, however, Raman scatter fewer than one in a million incident photons, with far more photons undergoing elastic scattering. In order to detect the weak Raman signal, the elastic scatter must be spectrally filtered from the Raman light, and is usually discarded. The spatial distribution of elastically scattered light from a target, however, contains useful information about its morphological structure.

Several researchers have recently used the angular distribution of elastically scattered light for biological applications such as distinguishing between cancerous and non-cancerous tissues $[2,3]$, analyzing the effects of photodynamic therapy on cancer cells $[4,5]$, and observing calcium-dependent changes in mitochondrial morphology [6]. The most common approach is a planar or quasi-planar wave illumination scheme, as the scattering can then be analyzed by well-established Mie theory to extract the mean radius of a population of scatterers by a best-fit metric. $[4,7]$ In these situations, to approximate planar illumination locally, the illumination spot diameter is on the order of .5 to several millimeters. 
More recent extensions of Mie theory, describing scattering from Gaussian beams, imply that it should be possible to extract size information using the focused laser beams required for Raman micro-spectroscopy, even though the wavefronts have significant angular content [8]. One example of these extensions is Generalized Lorenz-Mie theory (GLMT) [9]. Briefly, Mie theory may be described as an eigenfunction problem where a plane wave is decomposed into a basis set of associated Legendre functions, and each of these basis functions is scattered by a sphere with different amplitudes depending on the radius of the sphere and its refractive index relative to the surrounding medium. [10] GLMT decomposes non-planar wavefronts in the spherical basis set in the same manner, yielding different weights for each term in the infinite sum of basis functions. In the general case of a scatterer removed from the beam waist in $x, y$, and $z$, the weights are laborious to compute, and are given in detail by Lock. [11] However, in the special case of a sphere centered in the Gaussian beam the weights are of the same order of complexity as in the traditional Mie theory case, and are given explicitly by Grehan et al. [9] This case was assumed in the analysis below.

We modified a home-built epi-Raman microscope to add an angular scattering channel as shown schematically in Figure 1. A 785 nm laser source ( $\mu$ Laser Systems, Garden Grove, CA) with a linearly polarized, single spatial mode output is coupled via a dichroic beamsplitter (Semrock, Rochester, NY) into a Nikon inverted microscope equipped with a 60x water immersion objective $(\mathrm{NA}=1.0)$. The incident light under-fills the back-aperture of the microscope objective by a factor of 6 in order to obtain an approximately $6 \mu \mathrm{m}$ Gaussian beam waist in the sample plane. This spot diameter was chosen to be slightly larger than the bead sizes discussed in this paper, but the spot size may be freely adjusted to suit the needs of other applications. In the Raman arm of the collection system, back-scattered, Stokes-shifted light is passed by the dichroic element and a holographic notch filter (Kaiser, Ann Arbor, MI), focused onto an optical fiber, and introduced into an imaging spectrograph and CCD array (Andor, Belfast, Northern Ireland). To provide an elastic scattering channel, a polarization-insensitive Fresnel beam-sampler pair inserted prior to the dichroic element picks off . $16 \%$ of the return light. A lens placed in this path relays the microscope objective's back focal plane to a second CCD array (Andor, Belfast, Northern Ireland), obtaining a twodimensional scattergram that Fourier-maps angular scattering intensity at different polar $(\theta)$ and azimuthal $(\phi)$ angles into different spatial locations in the image. This mapping is identical to that employed by other elastic scattering microscopes [5].

Polystyrene beads (Duke Scientific, Fremont, CA) were suspended in water and placed in a chamber constructed of a disc of 10 mil thick FEP Teflonß (Integument, Tonawanda, NY) held in a metal coverslip holder (Molecular Probes, Carlsbad, CA), shown in the inset to Figure 1. The Teflon $\AA$ was chosen for its optical clarity and close index match to water $(\mathrm{n}=1.343$, manufacturer specification), ensuring that the bead is surrounded by a nearly 
uniform refractive index. In the sample plane individual beads resting on the Teflon $\mathbb{R}$ were centered by hand within the laser beam focus, and illuminated with $10 \mathrm{~mJ}$ for the elastic scattering measurements.

Scattering data from this system and associated analysis are shown in Figure 2. Figures 2 (a), (c), (e), and (g) show noticeably different experimental scattergrams obtained from 4 different single polystyrene beads. Figure 2 (i) shows a one-dimensional log-scale cutthrough of the two-dimensional data shown in Figure 2 (c), corresponding to the angular range $\theta=192^{\circ}$ to $219^{\circ}$ at $\phi=0$, indicated by the white line. The angular range $\theta=180^{\circ}$ to $192^{\circ}$ is obscured by a direct back reflection from the microscope objective, while angles above $219^{\circ}$ are outside the collection cone defined by the microscope objective's numerical aperture. Several 1-d slices through the data (at $\phi=0,45,90,135)$ were compared to theory curves calculated for sphere diameters ranging from 1 to 12 microns, with $20 \mathrm{~nm}$ spacing, with goodness of fit being determined by a standard reduced $\chi^{2}$ metric [12]. To emphasize the sensitivity of the fit to sphere diameter, values of $1 / \chi^{2}$ for the bead of Figure 2 (c) are shown in Figure $2(\mathrm{j})$. There is a clear maximum, corresponding to a best-fit diameter, at $\mathrm{d}=4.18 \mu \mathrm{m}$. The full GLMT prediction for all $\theta$ and $\phi$ values, shown in Figure $2(\mathrm{~d})$, reproduces all of the major features of the experimental data. To test the repeatability of the elastic scattering measurements against noise and slight changes in alignment, a single bead was moved in and out of the beam and measured a total of 10 independent times. $\chi^{2}$ optimization yielded identical results for all 10 measurements within the $20 \mathrm{~nm}$ resolution set by the spacing between calculated theory curves. The analysis was then repeated using more finely spaced theory curves centered at the best-fit point from the coarsely spaced analysis (60 $\mathrm{nm}$ on either side of the best-fit point, with $1 \mathrm{~nm}$ spacing). With the more finely spaced analysis, the standard deviation of the predicted diameters was $3 \mathrm{~nm}$. The predicted diameter returned by our analysis was only weakly sensitive to refractive index fluctuations in the second decimal place, so our final analysis assumed the manufacturer specified refractive index value for the polystyrene spheres $(\mathrm{n}=1.59)$. In cases where the refractive index is not known a priori, such as for organelles within a cell, this parameter can be fit simultaneously [5].

This same 2-step $\chi^{2}$ analysis was performed on the other three beads, producing the bestfit scattergrams shown in Figures 2 (b), (f), and (h), again reproducing all of the major experimental features. The four beads' predicted diameters of 4.266, 4.190, 2.019, and 5.011 $\pm .003 \mu \mathrm{m}$ are all plausible. Beads (a), (c), and (g) were selected from a distribution of spheres with a quoted mean diameter of $5 \mu \mathrm{m} \pm .3 \mu \mathrm{m}$, while bead (e) is from a $2 \mu \mathrm{m} \pm$ $0.02 \mu \mathrm{m}$ distribution. Although the predicted sizes for the beads in Figures 2 (a) and (c) are beyond $2 \sigma$ of the manufacturer's distributions, estimates of the beads' diameters based on captured white light microscope images revealed them to be closer to $4 \mu \mathrm{m}$ than $5 \mu \mathrm{m}$ 
in size, unlike other beads studied from the same population. Obtaining scattergrams from individual outliers is one of the advantages of being able to size single particles. Sensitivity to bead size is most evident in comparing Figures 2 (a) and (c), where the scattergrams are visibly different but are well-modeled using diameters that differ by only $80 \mathrm{~nm}$. The scattergram in Figure 2 (e), meanwhile, is radically different from the other three patterns, corresponding to its originating from a substantially smaller bead.

The system is also capable of taking simultaneous Raman spectra of the scattering target, as demonstrated by the Raman spectrum of a single polystyrene bead shown in Figure 2 $(\mathrm{k})$. The total delivered energy to the bead for the Raman acquisition was approximately $100 \mathrm{~mJ}$, starting simultaneously with the elastic scattering acquisition but continuing for 10 times longer to improve signal to noise. The spectrum has been background corrected to remove the spectral contributions from the Teflon $\cap$ substrate, water, and the microscope objective. The Raman spectrum can be analyzed to determine chemical components and mode vibrations. For example, the peak at $1003 \mathrm{~cm}^{-1}$ is attributed to the $\nu_{12}$ out-of-plane bending mode of polystyrene's carbon rings [13].

Currently, fits of experiment to theory using traditional Mie theory are about as GLMT fits, given the present experimental conditions, bead radius, and beam waist size. However, this would not be the case if system noise were improved, if the beam was focused more tightly, or if the bead more totally filled the beam waist. As noted already, the scattergram in Figure $2(\mathrm{~h})$ is the GLMT model for the best-fit diameter ( $\mathrm{d}=5.011$ microns). The pattern in Figure 2 (l), which depicts the Mie theory prediction for a bead of the same diameter, exhibits a simpler pattern of rings that bears less resemblance to the measured data.

In conclusion, the results presented here demonstrate that collecting the elastically scattered light in a traditional Raman microscope yields an extra dimension of information with no modifications to the illumination system beyond what is needed for a normal Raman microscope. Using both collection channels, the target can now be characterized both chemically and morphologically. Following this validation on the test case of polystyrene beads, future experiments are planned for single cells. Preliminary results indicate that such scattergrams, while noticeably different between cell types, contain fixed speckle patterns. Because this is not seen in Mie-scattering studies using collimated beams and many cells, we tentatively attribute the speckle to coherent interferences among the relatively smaller number of contributing scatterers, whose positions are not changing appreciably during the measurement. [14] This effect could be mitigated, however, by making multiple measurements from the same cell while slightly varying either the cell position or the beam properties. By averaging these measurements together, the amplitude of coherent oscillations in the final pattern should be reduced, permitting the traditional assumption that the total pattern of multiple scatterers is an incoherent sum of individual scattering patterns. 
The authors wish to thank Thomas Foster and Thomas Brown for their many helpful and illuminating discussions. This work was supported in part by NIH grant 1-R21-DE016111$01 \mathrm{~A} 1$. 


\section{References}

1. M. Mannie, T. McConnell, C. Xie, and Y.-Q. Li, "Activation-dependent phases of t cells distinguished by use of optical tweezers and near infrared Raman spectroscopy," Journal of Immunological Methods 297 (2005).

2. J. Mourant, T. Johnson, S. Carpenter, A. Guerra, T. Aida, and J. Freyer, "Polarized angular dependent spectroscopy of epithelial cells and epithelial cell nuclei to determine the size scale of scattering structures," Journal of Biomedical Optics 7, 378-387 (2002).

3. J. Pyhtila and A. Wax, "Rapid, depth-resolved light scattering measurements using Fourier domain, angle-resolved low coherence interferometry," Optics Express 12, 6178$6183(2004)$.

4. J. Wilson, C. Bigelow, D. Calkins, and T. Foster, "Light scattering from intact cells reports oxidative-stress-induced mitochondrial swelling," Biophysical Journal 88, 29292938 (2005).

5. W. Cottrell, J. Wilson, and T. Foster, "Microscope enabling multimodality imaging, angle-resolved scattering, and scattering spectroscopy," Optics Letters 32, 2348-2350 (2007).

6. N. Boustany, R. Drezek, and N. Thakor, "Calcium-induced alterations in mitochondrial morphology quantified in situ with optical scatter imaging," Biophysical Journal 83, 1691-1700 (2002).

7. A. Wax, C. Yang, V. Backman, M. Kalashnikov, R. Dasari, and M. Feld, "Determination of particle size by using the angular distribution of backscattered light as measured with low-coherence interferometry," Journal of the Optical Society of America A 19, 737-744 (2002).

8. H. Fang, L. Qiu, E. Vitkin, M. Zaman, C. Andersson, S. Salahuddin, L. Kimerer, P. Cipolloni, M. Modell, B. Turner, S. Keates, I. Bigio, I. Itzkan, S. Freedman, R. Bansil, E. Hanlon, and L. Perelman, "Confocal light absorption and scattering spectroscopic (class) microscopy," Applied Optics 46, 1760-1769 (2007).

9. G. Grehan, B. Maheu, and G. Gouesbet, "Scattering of laser beams by Mie scatter centers: Numerical results using a localized approximation," Applied Optics 25, 35393548 (1986).

10. C. Bohren and D. Huffman, Absorption and Scattering by Small Particles (Wiley Interscience, New York, NY, 1983).

11. J. Lock, "Improved Gaussian beam-scattering algorithm," Applied Optics 34, 559-570 (1995).

12. P. Bevington, Data Reduction and Error Analysis for the Physical Sciences (McGrawHill, New York, NY, 1969). 
13. B. Jasse, R. Chao, and L. Koenig, "Laser Raman scattering in uniaxially oriented atactic polystyrene," Journal of Polymer Science: Polymer Physics Edition 16, 2157-2169 (1978).

14. C. Xu, P. Carney, and S. Boppart, "Wavelength-dependent scattering in spectroscopic optical coherence tomography," Optics Express 13, 5450-5462 (2005). 


\section{List of Figures}

1 Schematic diagram of IRAM system. $\mathrm{L}=785 \mathrm{~nm}$ laser source, FBS $=$ Fresnel beam-sampler, DBS = dichroic beam-splitter, HNF = holographic notch filter, $\mathrm{NDF}=$ neutral density filter, $\mathcal{O}$ and $\mathcal{O}^{\prime}$ are the object and image planes, respectively, and $\mathcal{F}$ and $\mathcal{F}^{\prime}$ are the microscope objective's Fourier plane and its image. Inset shows water immersion objective in contact with indexed matched teflon, with bead in chamber, suspended in water. . . . . . . .

2 (color online) (a-h) Experimental scattergrams and associated best-fit theoretical models using GLMT from 4.266(a,b), 4.190(c,d), 2.019(e,f), and 5.011(g,h) micron polystyrene beads. Grey scale runs from black low to white high (blue low to red high online). The artifacts in the center of the experimental data are caused by an intense back-reflection from the microscope objective. (i) $\phi=0^{\circ}$ cut-through of 2 dimensional data from scattergram (c) and theoretical fit for 4.190 micron bead. (j) $1 / \chi^{2}$ surface showing a strong peak at 4.18 $\mu \mathrm{m}$ which was later refined to $4.190 \mu \mathrm{m}(\mathrm{k})$ Raman scattering spectrum from a polystyrene bead gathered simultaneously with elastic scattering data. (l) Mie theory scattergram for a $5.011 \mu \mathrm{m}$ bead. . . . . . . . . . . . . . . 10 


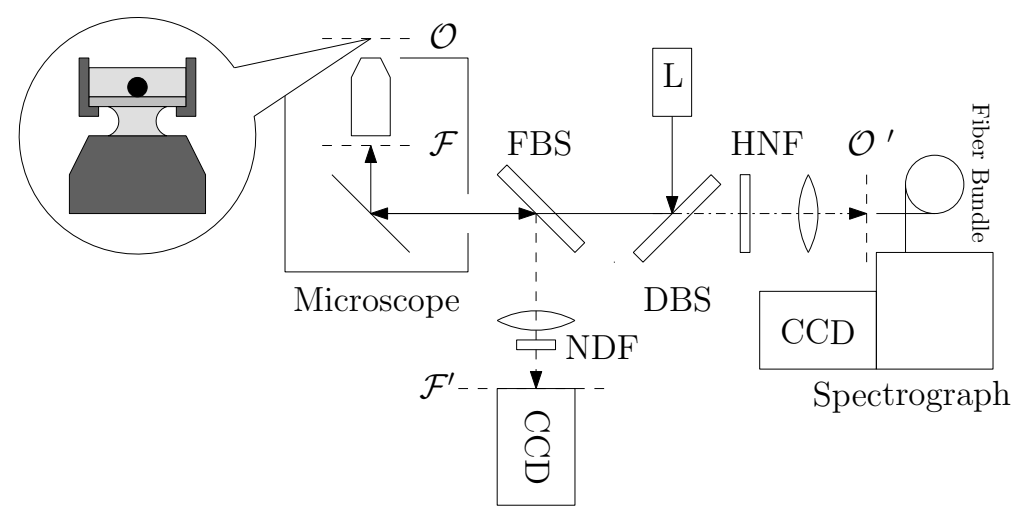

Fig. 1. Schematic diagram of IRAM system. $\mathrm{L}=785 \mathrm{~nm}$ laser source, FBS $=$ Fresnel beam-sampler, DBS = dichroic beam-splitter, HNF = holographic notch filter, $\mathrm{NDF}=$ neutral density filter, $\mathcal{O}$ and $\mathcal{O}^{\prime}$ are the object and image planes, respectively, and $\mathcal{F}$ and $\mathcal{F}^{\prime}$ are the microscope objective's Fourier plane and its image. Inset shows water immersion objective in contact with indexed matched teflon, with bead in chamber, suspended in water. 

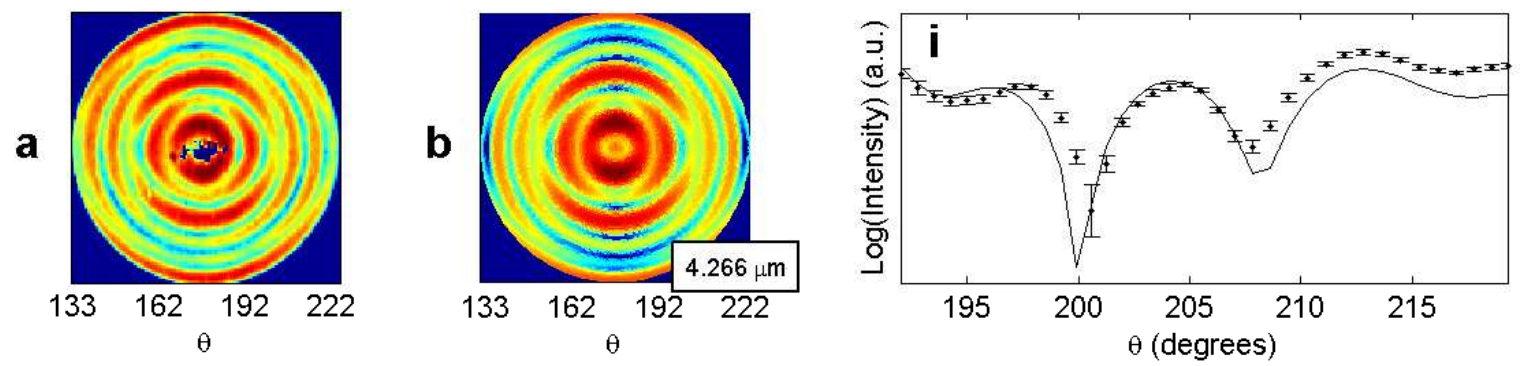

C
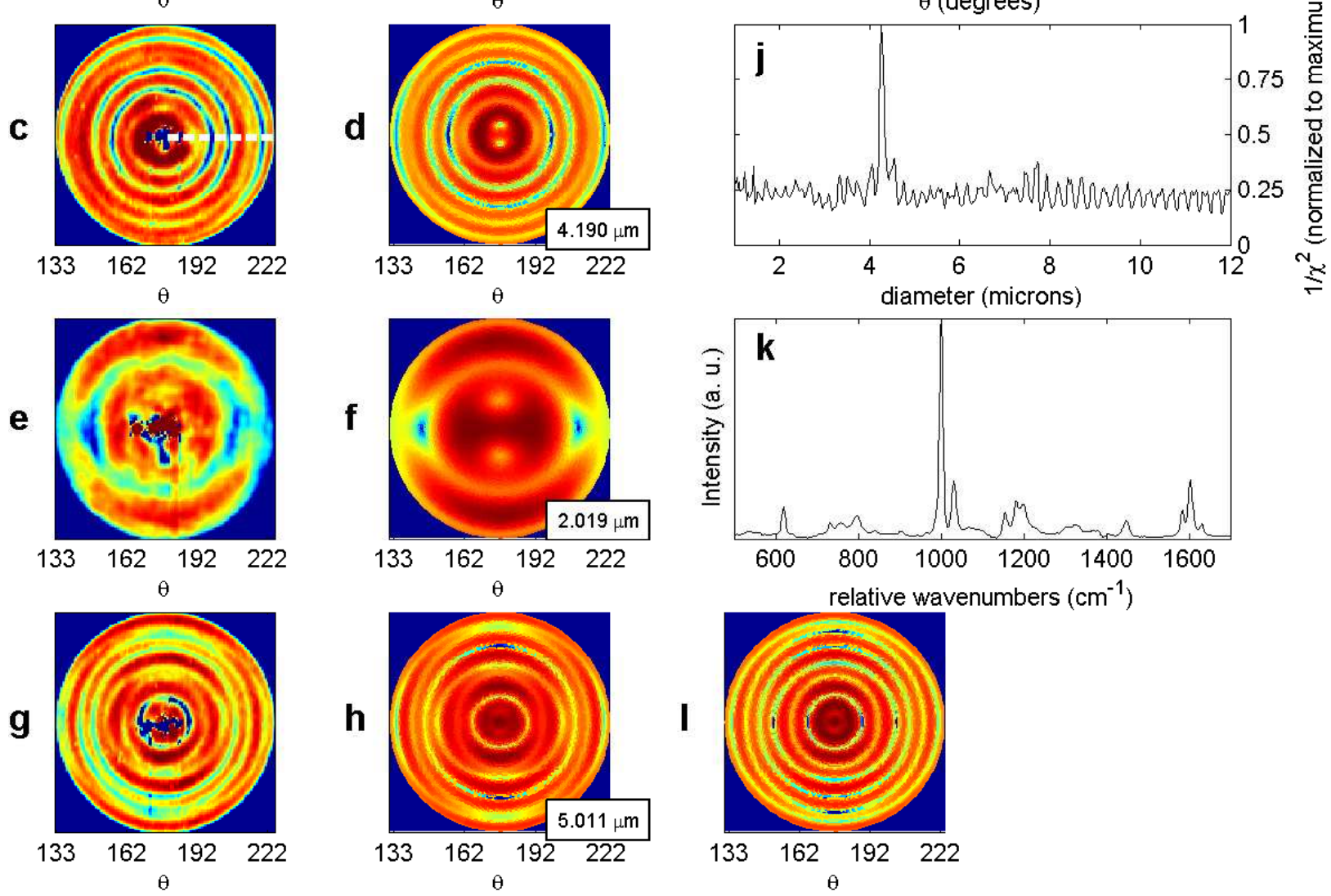

Fig. 2. (color online) (a-h) Experimental scattergrams and associated best-fit theoretical models using GLMT from 4.266(a,b), 4.190(c,d), 2.019(e,f), and $5.011(\mathrm{~g}, \mathrm{~h})$ micron polystyrene beads. Grey scale runs from black low to white high (blue low to red high online). The artifacts in the center of the experimental data are caused by an intense back-reflection from the microscope objective. (i) $\phi=0^{\circ}$ cut-through of 2 dimensional data from scattergram (c) and theoretical fit for 4.190 micron bead. (j) $1 / \chi^{2}$ surface showing a strong peak at $4.18 \mu \mathrm{m}$ which was later refined to $4.190 \mu \mathrm{m}(\mathrm{k})$ Raman scattering spectrum from a polystyrene bead gathered simultaneously with elastic scattering data. (1) Mie theory scattergram for a $5.011 \mu \mathrm{m}$ bead. 